\title{
RULED SUBMANIFOLDS OF FINITE TYPE
}

\author{
FRANKI DILLEN
}

(Communicated by Jonathan M. Rosenberg)

\begin{abstract}
We show that a ruled submanifold of finite type in a Euclidean space is a cylinder on a curve of finite type or a generalized helicoid.
\end{abstract}

\section{INTRODUCTION}

The well-known theorem of Catalan states that the only ruled minimal surfaces in Euclidean 3-space are the plane and the helicoid. This classical result can be generalized in two directions.

First one can say that a submanifold $M^{n+1}$ of a Riemannian manifold $M^{n+p}$ is ruled, if $M^{n+1}$ is foliated by $n$-dimensional totally geodesic subspaces of $M^{n+p}$, and one can try to classify ruled minimal submanifolds of a Euclidean space. This is done independently by Ü. Lumiste in [7], by C. Thas in [9], and by J. M. Barbosa, M. Dacjzer, and L. P. Jorge in [2]. They show that a minimal ruled submanifold of a Euclidean space is a generalized helicoid. More details will be given in $\S 1$. This extends the theorem of Catalan in one direction.

On the other hand, minimal submanifolds of a Euclidean space can be considered as a special case of submanifolds of finite type. Submanifolds of finite type are introduced by B.-Y. Chen in [4]. A submanifold $M^{n}$ of a Euclidean space $\mathbf{E}^{n+p}$ is said to be of finite type if each component of its position vector field $X$ can be written as a finite sum of eigenfunctions of the Laplacian $\Delta$ of $M^{n}$, i.e. if $X=X_{0}+X_{1}+\cdots+X_{k}$, where $X_{0}$ is a constant vector and $\Delta X_{i}=\lambda_{i} X_{i}$ for $i=1, \ldots, k$. If in particular all eigenvalues $\left\{\lambda_{1}, \lambda_{2}, \ldots, \lambda_{k}\right\}$ are mutually different, then $M^{n}$ is said to be of $k$-type. Note that every minimal submanifold of a Euclidean space is of 1-type, since $\Delta X=0$.

In [5] the Catalan theorem was extended to surfaces of finite type in a Euclidean space $\mathbf{E}^{n}$. There it is proved that a ruled surface of finite type in a Euclidean space is either a part of a cylinder over a curve of finite type or a helicoid in $\mathbf{E}^{3}$. In particular it follows that a ruled surface of finite type in $\mathbf{E}^{3}$ is a part of a plane, a circular cylinder or a helicoid.

In this paper we extend the Catalan theorem in both directions at the same time by showing the following theorem.

Received by the editors October 4, 1990.

1980 Mathematics Subject Classification (1985 Revision). Primary 53A07, 53B25.

Key words and phrases. Finite type submanifolds, ruled submanifolds, generalized helicoid.

Supported by a post-doctoral fellowship of the Research Council of the Katholieke Universiteit Leuven. 
Theorem. A ruled submanifold $M^{n+1}$ in $\mathbf{E}^{n+p}$ is of finite type if and only if $M^{n+1}$ is a part of a cylinder on a curve of finite type or a part of a generalized helicoid.

When restricting to hypersurfaces, we have the following corollary.

Corollary. A ruled hypersurface $M^{n+1}$ in $\mathbf{E}^{n+2}$ is of finite type if and only if $M^{n+1}$ is a part of a hyperplane, a circular cylinder, a helicoid $H^{2}$ in $\mathbf{E}^{3}$, a cone $H^{3}$ with top $P$ on a minimal ruled surface in a 3-sphere, centered at $P$, or a cylinder on $H^{2}$ or $H^{3}$.

\section{RULED MiNimal SUBMANIFoldS OF A EUCLIDEAN SPACE}

In 1958 Ü. Lumiste showed in [7] that a minimal ruled submanifold $M^{n+1}$ of a Euclidean space is either

(i) generated by an $n$-dimensional affine subspace $P$ under a screw motion in $\mathbf{E}^{2 n+1}$ such that the axis cuts $P$ orthogonal,

(ii) generated by an $n$-dimensional affine subspace $P$ under a rotation in $\mathbf{E}^{2 n}$ around a point in $P$,

(iii) a cylinder on a submanifold of the type (i) or (ii).

Analytically any minimal submanifold therefore can be given by

$$
\begin{array}{r}
X\left(s, t_{1}, \ldots, t_{n}\right)=\left(t_{1} \cos \left(a_{1} s\right), t_{1} \sin \left(a_{1} s\right), \ldots,\right. \\
\left.\quad t_{k} \cos \left(a_{k} s\right), t_{k} \sin \left(a_{k} s\right), t_{k+1}, \ldots, t_{n}, b s\right),
\end{array}
$$

where $a_{1}, \ldots, a_{k}$ and $b$ are real numbers. A submanifold with this kind of parameterization is called a generalized helicoid. If $b \neq 0$, resp. $b=0$, then this gives a cylinder on a submanifold of the type (i), resp. (ii). This analytic description was given independently by C. Thas in [9] in 1979, who proved directly that any minimal ruled submanifold can be parameterized by $(*)$. In 1984 this result was proved again by J. M. Barbosa, M. Dacjzer, and L. P. Jorge in [2]. They show that any minimal ruled submanifold is generated by an affine subspace $P$ under a one-parameter subgroup $A$ of rigid motions of the Euclidean space, such that $P$ is orthogonal to the orbits of $A$. Then they show that the resulting submanifold (at least if it is minimal) has a parameterization $(*)$. They also extend tineir result to ruled submanifolds of real space forms.

I would like to point out that a ruled submanifold of the type (ii) is a cone on a minimal ruled submanifold of some hypersphere of the Euclidean space. Indeed, putting $k=n$ and $b=0$ in $(*)$ gives that $M^{n+1}$ is a cone with as top the origin of $\mathbf{E}^{2 n}$. In particular, the rulings of $M^{n+1}$ pass through the origin. Then the intersection of $M^{n+1}$ and a sphere $S^{2 n-1}$, centered at the origin, is a ruled submanifold $M^{\prime}$ in $S^{2 n-1}$. Using the fact that a submanifold $M^{\prime}$ of a sphere $S^{m}$ is minimal (in the sphere) if and only if the cone shaped on $M^{\prime}$ with as top the center of the sphere is minimal in $\mathbf{E}^{m+1}$, cf. [8, Proposition 6.1.1], we obtain that $M^{\prime}$ is minimal in $S^{2 n-1}$. Note that this argumentation reduces the classification of minimal ruled submanifolds of a sphere to the classification of minimal ruled submanifolds of the Euclidean space whose rulings pass through a fixed point.

Finally, if we look for ruled minimal hypersurfaces $M^{n+1}$ of a Euclidean space $\mathbf{E}^{n+2}$, we see that there are essentially (up to cylinders, built on those submanifolds) three possibilities, namely $M^{n+1}$ is of the type (i) and $n=1$, in 
which case we obtain a helicoid in $\mathbf{E}^{3}$, or $M^{n+1}$ is of the type (ii) and $n=1$, in which case we obtain a plane, or $M^{n+1}$ is of the type (ii) and $n=2$, in which case we obtain cone (with top 0 ) on a minimal ruled surface in a 3dimensional sphere, centered at 0 . We remark that ruled minimal surfaces in a 3-sphere were classified by H. B. Lawson in [6], that a classification of ruled minimal hypersurfaces of a Euclidean space was also given by G. Aumann in [1], and that complete minimal hypersurfaces of a Euclidean space were classified by D. Blair and J. R. Vanstone in [3].

\section{RULED SUBMANIFOLDS OF FINITE TYPE}

Let $M^{n+1}$ be a ruled submanifold of $\mathbf{E}^{n+p}$. Let $\alpha$ be an orthogonal trajectory of $M^{n+1}$ and assume $\alpha$ to be parameterized by arc length. Let $\left\{e_{1}(s), \ldots\right.$, $\left.e_{n}(s)\right\}$ be a set of orthonormal vector fields along $\alpha$ such that $\left\{e_{1}(s), \ldots, e_{n}(s)\right\}$ span the ruling of $M^{n+1}$ through $\alpha(s)$. The set $\left\{e_{1}(s), \ldots, e_{n}(s)\right\}$ can be chosen such that $\left\langle e_{i}^{\prime}(s), e_{j}(s)\right\rangle=0$ for all $i$ and $j$, see [2, Lemma 2.2]. So we can give a parameterization of $M^{n+1}$ by

$$
X\left(s, t_{1}, \ldots, t_{n}\right)=\alpha(s)+\sum_{i=1}^{n} t_{i} e_{i}
$$

If we define a function $q$ on $M^{n+1}$ by

$$
q=\left\|X_{s}\right\|^{2}=1+2 \sum_{i=1}^{n} t_{i}\left\langle\alpha^{\prime}, e_{i}^{\prime}\right\rangle+\sum_{i, j=1}^{n} t_{i} t_{j}\left\langle e_{i}^{\prime}, e_{j}^{\prime}\right\rangle,
$$

then the Laplacian $\Delta$ of $M^{n+1}$ can be expressed as follows:

$$
\Delta=-\sum_{i=1}^{n} \frac{\partial^{2}}{\partial t_{i}^{2}}-\frac{1}{q} \frac{\partial^{2}}{\partial s^{2}}+\frac{1}{2} \frac{\partial q}{\partial s} \frac{1}{q^{2}} \frac{\partial}{\partial s}-\frac{1}{2} \frac{1}{q} \sum_{i=1}^{n} \frac{\partial q}{\partial t_{i}} \frac{\partial}{\partial t_{i}}
$$

Note that $q$ is a polynomial in $t=\left(t_{1}, \ldots, t_{n}\right)$ with functions in $s$ as coefficients. The degree of $q$ is 2 , unless all $e_{i}^{\prime}$ are zero. If all $e_{i}^{\prime}$ are identically zero, then $M^{n+1}$ is a cylinder. If $M^{n+1}$ is a cylinder, then, similarly as in [5], one can show that $M^{n+1}$ is a cylinder on a curve of finite type. So from now on we assume that $M^{n+1}$ is not cylindrical. Then we can assume, by restricting to a sufficiently small piece of $\alpha$, that $q$ has degree 2 everywhere. The proof of the following lemma is straightforward.

Lemma. If $P$ is a polynomial in $t=\left(t_{1}, \ldots, t_{n}\right)$ with functions in $s$ as coeffcients and $\operatorname{deg}(P)=d$, then

$$
\Delta\left(\frac{P(t)}{q^{m}}\right)=\frac{\widetilde{P}(t)}{q^{m+3}}
$$

where $\widetilde{P}$ is a polynomial in $t$ with functions in $s$ as coefficients and $\operatorname{deg}(\widetilde{P}) \leq$ $d+4$.

The theorem now can be proved similarly as in [5]. Since the argumentation is short, we repeat it here. If $M^{n+1}$ is of $k$-type, it follows, cf. [4, p. 256], that there exist numbers $c_{1}, \ldots, c_{k}$, such that

$$
\Delta^{k+1} X+c_{1} \Delta^{k} X+\cdots+c_{k} \Delta X=0 .
$$


Let $X_{i}$ be any component of $X$. We know that $X_{i}$ is a linear function in $t$ with functions in $s$ as coefficients. By applying the lemma, we easily obtain that

$$
\Delta^{r} X_{i}=\frac{P_{r}(t)}{q^{3 r-1}} \quad \text { and } \quad \operatorname{deg}\left(P_{r}\right) \leq 1+4 r
$$

Hence if $r$ goes up by one, the degree of the numerator of $\Delta^{r} X_{i}$ goes up by at most 4 , while the degree of the denominator goes up by 6 . Hence the sum $(* *)$ can never be zero, unless $\Delta X_{i}=0$. Therefore $M^{n+1}$ is minimal, so that $M^{n+1}$ is a generalized helicoid.

In order to prove the corollary, it is sufficient to remark that a plane curve of finite type is a part of a circle or a straight line [5, Theorem 3].

\section{REFERENCES}

1. G. Aumann, Die Minimalhyperregelflächen, Manuscripta Math. 34 (1981), 293-304.

2. J. M. Barbosa, M. Dajczer, and L. P. Jorge, Minimal ruled submanifolds in spaces of constant curvature, Indiana Univ. Math. J. 33 (1984), 531-547.

3. D. Blair and J. R. Vanstone, A generalization of the helicoid, Minimal Submanifolds and Geodesics, Kaigai Publications, Tokyo, 1978, pp. 13-16.

4. B.-Y. Chen, Total mean curvature and submanifolds of finite type, World Scientific, Singapore, 1984.

5. B.-Y. Chen, F. Dillen, L. Verstraelen, and L. Vrancken, Ruled surfaces of finite type, Bull. Austral. Math. Soc. 42 (1990), 447-453.

6. H. B. Lawson, Jr., Complete minimal surfaces in $S^{3}$, Ann. of Math. (2) 92 (1970), 335-374.

7. Ü. Lumiste, Die $n$-dimensionalen Minimalflächen mit einer $(n-1)$-dimensionalen asymptotischen Richtung im jedem Punkte, Tartu Riikl. Ül. Toimetised 62 (1958), 117-141. (Russian, German summary)

8. J. Simons, Minimal varieties in riemannian manifolds, Ann. of Math. (2) 8 (1968), 62-105.

9. C. Thas, Minimal generalized ruled surfaces in the Euclidean space $\mathbf{E}^{m}$, Internal Report Seminar of Higher Geometry, State University of Ghent.

Katholieke Universiteit Leuven, Departement Wiskunde, Celestijnenlaan 200 B, B3001 LeUVEN, BelgiUm

E-mail address: fgaba01@blekul11.bitnet 